\title{
SCALABILITY CONCERNS OF CHIRP SPREAD SPECTRUM FOR LPWAN APPLICATIONS
}

\author{
Aiju Thomas ${ }^{1}$ and N V Eldhose ${ }^{2}$ \\ ${ }^{1}$ School of Technology and Applied Science, M G University, Kottayam, India \\ ${ }^{2}$ M G University, Kottayam, India
}

\begin{abstract}
Divergent modulation schemes have been proposed for the Internet of Things (IoT). Low Power Wide Area Networks (LPWAN) technologies are gaining unprecedented acceptance in IoT application of sensor networks. Chirp Spread Spectrum (CSS) is a prominent modulation technique proposed for LPWAN. Chirps can traverse long distance and are resilient to noise and Doppler effects. Noise resilience along with transmission range and low power requirement makes CSS a preferred modulation scheme for sensor networks. LoRaWAN ${ }^{T M}$, with its physical (PHY) layer using CSS, has emerged as the widely accepted LPWAN solution. By using CSS modulation with orthogonal spreading factors (SF), LoRa offers wide coverage to LPWAN applications while supporting a high volume of devices. However, scalability performance of CSS has not been inadequately modeled. As with the suitability of the modulation scheme, there are concerns on how chirps interact with the surrounding as the number of deployments bursts out into higher volumes. We evaluate CSS at ISM band $868 \mathrm{MHz}$ for spreading factor 7 to 12 at bandwidth 125 $\mathrm{kHz}$ for performance and scalability. Simultaneous transmissions were simulated with repeated iterations and conclusions are arrived on collisions rate, packet error rate, and bit error. Suitability of using CSS for sensor networks for future deployments is commended.
\end{abstract}

\section{KEYWORDS}

LoRA, LPWAN, Sensor networks, CSS, Chirp Spread Spectrum, Packet error, collision

\section{INTRODUCTION}

Internet of Things (IoT) is a paradigm that creates a panoply of interconnected devices. Widespread deployment of IoT imposes complexity in communication and heterogeneity among devices which communicate over the Internet. The current deployments of things are in the order of 50 billion and by 2020 and will encompass 1000 times more connected devices, all with different requirements. There are concerns about how devices will interact with the surrounding and among themselves. This is however difficult to assume as today's network is limited with its ability to address the requirements of future deployments. The scalability of networks is a concern as to cope with the ever-growing number of devices [1]. Machine to Machine (M2M) communication as part of IoT is characterized by divergent modulation schemes. Modulation schemes are application specific, depends on the volume of data handled and range. A surveillance camera is required to deliver a huge volume of data, whereas applications like metering handle a small amount of data while being stationary. IoT applications such as sensor networks are often battery powered and require scalable low power wireless technologies. Low Power Wide Area Networks (LPWAN) has been proposed to address the needs of long-range and low-power IoT applications in the field of sensor networks.

Wireless sensor networks currently practice cellular-based technologies or multi-hop short-range technologies for LPWAN [2]. Devices or things use dedicated protocol stack to cope with the 
constraints of an end device. Constraints are set on power usage, range, computing capability, and bandwidth. In these networks, one of the things needs to connect to the internet through an internet protocol (IP) forming gateway whereas all the other form network among them in star or mesh topology. Applications and services are deployed at the top of the protocol stack which either runs locally or as part of the cloud.

There are standards that operate mostly at $2.4 \mathrm{GHz}$ and optionally in the $868 / 915 \mathrm{MHz}$, unlicensed frequency bands used to connect things. Prominent among them are

- Near Field Communications (NFC) for short range applications;

- Radio Frequency Identification (RFID) systems for active or passive networks;

- $\quad \mathrm{ZigBee}{ }^{\mathrm{TM}}, 6 \mathrm{LoWPAN}$, Thread-based systems under IEEE 802.15.4 standards;

- Bluetooth $^{\mathrm{TM}}$, Bluetooth Low Energy (BLE) ${ }^{\mathrm{TM}}$;

- IEEE 802.11 based $\mathrm{Wi}^{-\mathrm{Fi}^{\mathrm{TM}}}{ }^{\mathrm{TM}}$

Majority of these protocol use IEEE 802.15.4 based standard. These standards are weak in penetration capability and suffer heavy bandwidth interference. These technologies cannot be deployed for sensor networks due to range limitations. Another popular alternative is cellular networks connecting devices to the IoT gateway. The devices running on cellular networks use power-hungry algorithms for signal decoding. Deployments of such networks are expensive and the difficulty in device certification limits their application for sensor networks. Cellular networks are minimal in success addressing to LPWAN space despite its formidable presence. $2 \mathrm{G}$ cellular technologies are fading out: there are 30 million $2 \mathrm{G}$ base stations in the US alone which are to be orphaned [3]. 4G LTE or 5G with much more spectral efficiency is about to replace $2 \mathrm{G}$ technologies, leaving the carriers with no choice accepting consumer demand for high data speed. $4 \mathrm{G}$ and $5 \mathrm{G}$ networks cannot ensure scalability considering the volume of future deployment.

LPWAN which utilize the sub-GHz band and are can provide several kilometres of outdoor coverage. There have been efforts to reduce the bandwidth requirement for IoT devices. Driver encapsulation [4] techniques reduce bandwidth requirement hence enhance spectral efficiency of devices. The paper is organized in three sessions. Technologies related to LPWAN are discussed in session 2. Session 3 covers mathematical representation of CSS to aid simulation. Session 4 covers simulation environment and results.

\section{RELATED TECHNOLOGIES}

In order to help the reader better understand LPWAN, three prominent technologies supporting LPWAN for sensor networks are presented in this session.

\subsection{SIGFOX ${ }^{\mathrm{TM}}$}

Sigfox was proposed for the IoT market in 2009. They have a distinct market share since then. Sigfox operates in the $200 \mathrm{kHz}$ using the ultra narrow band modulation [3]. Each message is 100 $\mathrm{Hz}$ wide and transferring at 100 or 600 bits per second, depending on the region. Sigfox can achieve long range while being robust against noise. An uplink message has up to 12 bytes payload and takes an average $2 \mathrm{~s}$ over the air to reach the base stations which monitors the spectrum for Ultra Narrow Band (UNB) signals. For 12-byte data payload, a Sigfox frame uses 26 bytes in total [5]. The payload allowance in downlink messages is 8 bytes. A device is not attached to any specific base station like cellular networks. The broadcast messages are received by an average of three base stations in the range, and necessary approximations are made to 
decode the correct data. The first released technology supported only unidirectional uplink, however, bidirectional communication is now available [3]. Sigfox claims that each gateway can handle up to a million of connected objects, with a coverage area of $30-50 \mathrm{~km}$ in rural and 3-10 $\mathrm{km}$ in urban. But deployment to test the claim in terms of scalability is not achieved so far. Evaluation of network scalability cannot be performed as the technical details are kept unpublished as the protocol is proprietary in nature.

\subsection{INGENU ${ }^{\mathrm{TM}}$}

Ingenu is a preparatory LPWAN which has been around for Machine to Machine (M2M), Wireless Sensor Networking (WSN) and IoT [6]. Random Phase Multiple Access (RPMATM) uniquely addresses hundreds of IoT devices. RPMA was designed to provide secure LPWAN solutions at $2.4 \mathrm{GHz}$ band pioneering the 802.15.4k [7] standard. Despite the operating band at $2.4 \mathrm{GHz}$, the robustness of physical layer design can provide over long-range propagation under the most challenging RF environments.

\section{3. $\operatorname{LoRA}^{\mathrm{TM}}$}

LoRa is a proprietary spread spectrum modulation scheme which is a derivative of CSS. LoRa trades data rate for sensitivity within a fixed channel bandwidth [8]. It implements a variable data rate, utilizing orthogonal spreading factors, which allows the system designer to trade data rate for range or power and optimize network performance at a constant bandwidth. LoRa uses wideband linear frequency modulated pulses [9] whose frequency increases or decreases over a certain amount of time to encode information. This method will substantially increase receiver sensitivity due to the processing gain of the spread spectrum technique and exhibits a high tolerance to frequency misalignment between receiver and transmitter.

\section{Chirp Spread SPECTRUM}

Chirps are linear frequency modulated wideband pulses that are characterized by low power consumption, resilience to fading and Doppler effects. CSS combined with digital modulation schemes such as Binary Orthogonal Keying (BOK), Quadrature Phase Shift Keying (QPSK) and Differential Phase Shift Keying (DQPSK) can deliver better performance in Bit Error Rate (BER) [10]. Chirp Spread Spectrum (CSS) was initially proposed by Winkler for communication [11] and was applied to digital communication by Berni [12]. CSS is now being adopted for sensor networks due to its low power requirement, resilience to channel degradation by multipath fading and Doppler effects. IEEE 802.15.4 adopts CSS for Low Rate Wireless Personal Area Networks (LR - WPAN) for range and mobility. IEEE 802.15.4a standard [13] specifies CSS in two optional physical layers to support extended range.

- Ultra Wide Band (UWB) model, spanning from 2 to $10 \mathrm{GHz}$ frequency.

- UWB model for the frequency range from 100-1000 MHz

Narrowband frequency located within the mentioned range can be derived by a simple bandpass filtering. IEEE 802.11ah [14] is an extended range wireless protocol standard in sub-Gigahertz ISM band $(863-868 \mathrm{MHz}$ in Europe, $755-787 \mathrm{MHz}$ in China and $902-928 \mathrm{MHz}$ in North America) which minimize contention by extending their reach with low transmitted power. Radio propagation at these frequencies allows signals to travel greater distances, opens up opportunities to use with IoT. Semtech's LoRa ${ }^{\mathrm{TM}}$ [9] provides low power, low-cost long-range communication 
for IoT applications. LoRa ${ }^{\mathrm{TM}}$ utilize chirp spread spectrum for long-range communication. In LoRaWAN ${ }^{\mathrm{TM}}$ transceivers communicate with LoRa ${ }^{\mathrm{TM}}$ gateway which connects to the internet.

This paper examines LPWAN sensor networks in terms of Bit Error Ratio (BER), the number of packet collisions and packet error rate using MATLAB ${ }^{\mathrm{TM}}$. Evaluation is perfumed considering CSS as used LoRa physical layer. Modulated chirp signals were transmitted through an Additive White Gaussian Noise (AWGN) channel and evaluated for performance by varying Signal to Noise Ratios (SNR). Decoded signals are iterated manifold for consistency in results. A detailed mathematical model for CSS is presented in forthcoming sessions. Packet error rate and the number of collisions were analyzed with considerable numbers of sensors communicating simultaneously. Conclusions are drawn on scalability and range of CSS in LPWAN sensor networks.

\subsection{MATHEMATICAL REPRESENTATION OF CHIRPS}

\subsubsection{LINEAR CHIRPS}

Constant amplitude linear chirps are denoted as [6] [15] [9]

$$
x(t)=A \cos (\theta(t))
$$

where: $\theta(t)$ is the phase for a given time $t$

A is the amplitude of the chirp which is constant for a given chirp duration $\mathrm{T}$ sec outside which, A is zero.

Instantaneous frequency of the chirp can be represented as

$$
\omega(t)=\frac{d}{d t}(\theta(t))
$$

Time linearity of chirp can be achieved by making phase quadratic

$$
\theta(t)=2 \pi \mu r t^{2}+2 \pi f_{0} t+\phi
$$

Instantaneous angular frequency which is the derivative of phase $\theta(t)$ becomes

$$
\omega_{i}(t)=\frac{d}{d t} \theta(t)=4 \pi \mu t+2 \pi f_{0}
$$

Instantaneous frequency in $\mathrm{Hz}$ is given as

$$
f_{i}=2 \mu t+f_{0}
$$

In equation (5) frequency varies linearly with time with starting frequency at $f_{0}$. The rate of frequency change for chirp duration $\mathrm{T}$ secs is given as

$$
k=2 \mu t=\frac{\left(f_{1}-f_{0}\right)}{T}
$$


International Journal of Ad hoc, Sensor \& Ubiquitous Computing (IJASUC) Vol.10, No.1, February 2019

Sinusoidal sweeping frequency from $f_{0}$ to $f_{1}$ with phase as derivative $2 \pi\left(k t+f_{0}\right)$ is given as

$$
\cos \left(2 \pi \frac{k}{2} t^{2}+2 \pi f_{0} t\right)
$$

Linear chirp is represented in terms of time varying instantaneous frequency

$$
x(t)=A \cos (2 \pi f(t) t+\phi(t))
$$

where

$$
f(t)=\frac{k}{2} t+f_{0}
$$

In equation (2) if $\theta(t)$ is constant then frequency is zero, if $\theta(t)$ is linear then frequency is constant and if $\theta(t)$ is quadratic then frequency is linear.

\subsubsection{Chirp Spread Spectrum as used in LoRa}

LoRa utilizes CSS in its physical layer symbol modulation. Symbols are chirped using linear chirps. Chirps are spread to the entire spectrum of the carrier. CSS maintains constant timing and frequency offsets, reducing the complexity of receiver [9].

Spectral spreading is achieved by varying $\phi(t)$ of the chirp signal. Specific time function of raw chirp $f(t)$ exhibits linearity as

- Increases from $\frac{-b w}{2}$ to $\frac{b w}{2}$ for up chirps.

- Decreases from $\frac{b w}{2}$ to $\frac{-b w}{2}$ for down chirps.

Where: $b w$ is the spectral bandwidth in $\mathrm{Hz}$.

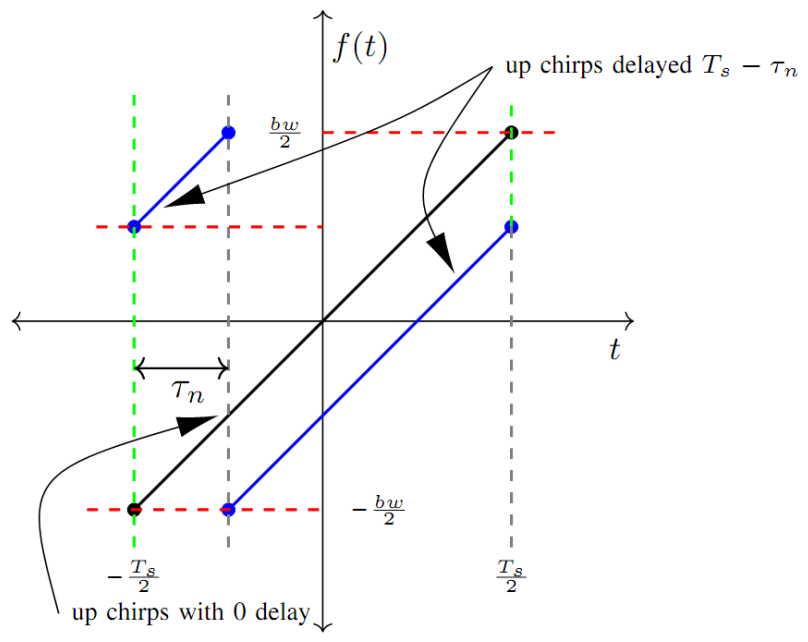

Figure 1. Chirp modulation of $n^{\text {th }}$ symbol 
$f(t)$ in terms of chirp duration $T_{s}$ and $b w$ is given as

Bandwidth and symbol period are related as

$$
f(t)= \pm \frac{b w}{T_{s}} t
$$

$$
T_{S}=\frac{2^{S F}}{b w} \operatorname{secs}
$$

Where $S F$ the spreading factor $\in[7,8,9,10,11,12]$

Symbol rate $R_{S}$ is given as

Data rate $R_{b}$ is derived as

$$
R_{S}=\frac{b w}{2^{S F}}=\frac{1}{T_{S}}
$$

$$
R_{b}=S F \frac{1}{\frac{2^{S F}}{b w}}=\frac{S F}{T_{S}}
$$

That is chirp rate $R_{b}$ which is equal to data rate is given as

$$
R_{b}=R_{S} 2^{S F} \text { chirps /sec }
$$

Equation 11 suggests that symbol rate R_s varies inversely with spreading factor SF. Noise resilience increases with enhanced SF. Thus by utilizing CSS with higher SF can enhance the range of transmission. Robustness of communication in terms of Bit Error Rate (BER) can be improved by using a suitable channel coding algorithm. Though LoRa protocol layers are kept unpublished, the possibility of using hamming code $(8,4)$ are revealed in many discussions.

Let us assume $\mathrm{N}$ set of symbols, chirp associated with any symbol $n, n \in[0, N-1]$ is unique. All the $\mathrm{N}$ chirps are orthogonal to each other and can be retrieved without intersymbol interference. Chirps for any symbol $n$ can be achieved by delaying $f(t)$ by

$$
\tau_{n}=\frac{n}{b w} \operatorname{secs}
$$

Figure 1 shows symbol $n$ modulated using up chirps delayed by $\tau_{n}$.

\section{Performance evaluation}

Remote deployed sensor networks are constrained in energy, reliability, processing capability and communication range. Majority of applications demand little or no direct human intervention. When thousands of sensors are deployed, frequent replacement of batteries itself is laborious [14]. In order to save power, these devices are kept in sleep mode and awakened upon alerted or scheduled. In this simulated version of CSS, preambles up chirps are transmitted by the remote machine which intends a communication to the network. Simple filter circuits trigger interrupt [15]. 


\subsection{SimULATION ENVIRONMENT}

An equivalent baseband model has been created for up and down chirps for error-free simulations of CSS. Modulated chirps were generated through linear phase variations keeping the amplitude constant satisfying equation (8). Eight preamble chirps indicate the beginning of package followed by two down chirps for synchronization. Symbols are generated from a binary combination of SF [16]. Each symbol is a unique chirp orthogonal to each other. In order to retrieve symbols at the receiver without inter symbol interference received signals are multiplied by orthogonal down chirps. In figure 1 symbol $\mathrm{n}$ is modulated as chirps from $\left[\frac{-T_{s}}{2}, \frac{T_{s}}{2}\right]$.

Chirps are spread as

$$
\begin{array}{lll}
f_{n(t)}=\frac{b w}{T_{S}}(t-\tau)+b w & \text { for } & t \in\left[-\frac{T_{S}}{2},-\frac{T_{S}}{2}+\frac{n}{b w}\right] \\
f_{n(t)}=\frac{b w}{T_{S}}(t-\tau) & \text { for } & t \in\left[-\frac{T_{S}}{2}+\frac{n}{b w}, \frac{T_{S}}{2}\right]
\end{array}
$$

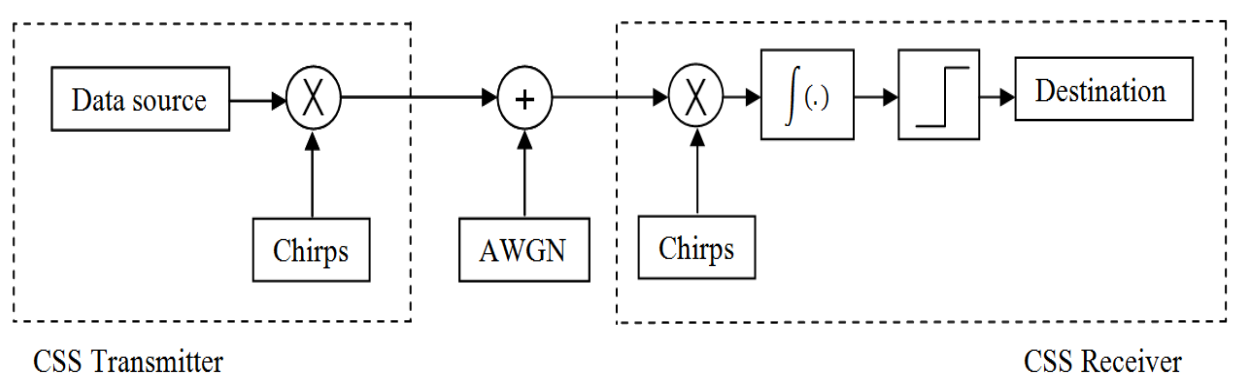

\subsection{SimUlation RESULTS}

Figure 2. Coding and decoding process of CSS

Figure 3 shows chirps generated for 100 symbols which are transmitted with 8 preambles up chirps and two synchronizing chirps. The generated chirps were transmitted through the AWGN channel with Signal to Noise Ratio (SNR) Odb. Received signals after band pass filtering were multiplied with orthogonal down chirps, to receive signal as shown in figure 4.

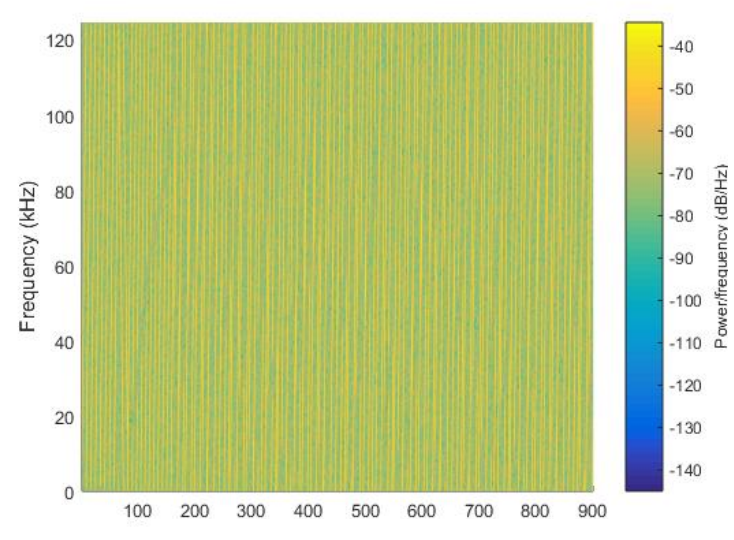

Figure 3.8 preamble, 2 sync and 100 symbols.

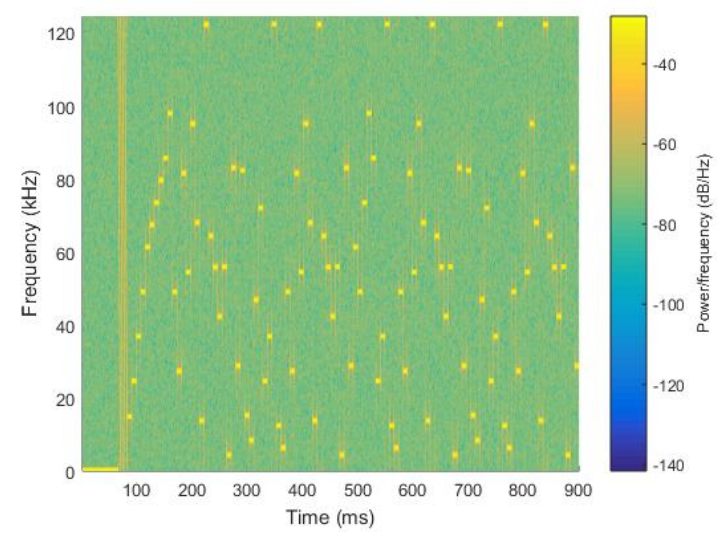

Fast Fourier Transform (FFT) performed on

Figure 4. After multiplication with orthogonal chirps at SNR Odb 
received signals which were transmitted through AWGN channel with SNR 0db and -20db are shown in figure 5 and figure 6 respectively.

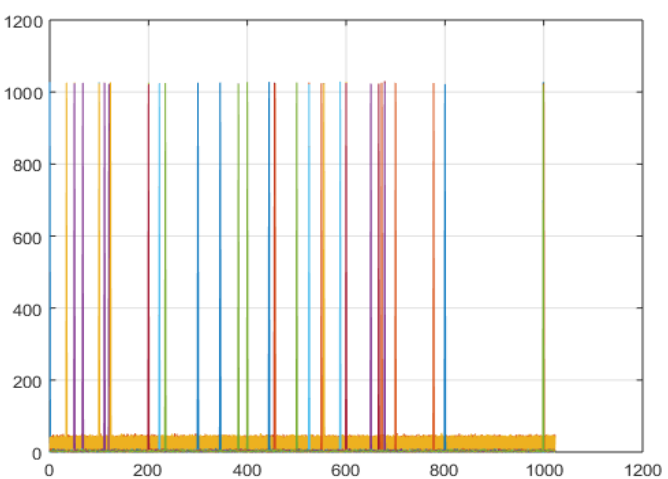

Figure 5. FFT performed after multiplying with orthogonal chirps (transmitted at SNR $=0 \mathrm{~dB}$ and $\mathrm{SF}=10)$

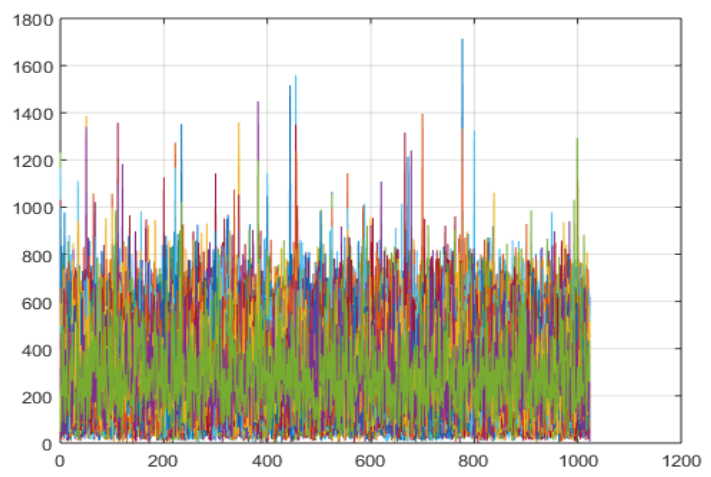

Figure 6. FFT performed after multiplying with orthogonal chirps (transmitted at SNR = $-20 \mathrm{~dB}$ and $\mathrm{SF}=10)$

On comparing FFT of spectrum received at SNR 0db and -20 dB, even though noise level is very high at $-20 \mathrm{~dB}$, with $\mathrm{SF}=10$, clarity in peaks corresponding to symbols are observed. Figure 7 shows the simulated performance plot of CSS prepared for SF $=7$ to 12, with Bandwidth (BW) and sampling frequency are selected as $125 \mathrm{kHz}$. With 8 preamble and 2 synchronization chirps, a total of 27720 bits were decoded after being sent through AWGN channel for SNR from -40db to 0db. Bit Error Rates (BERs) were computed for 100 iterations.

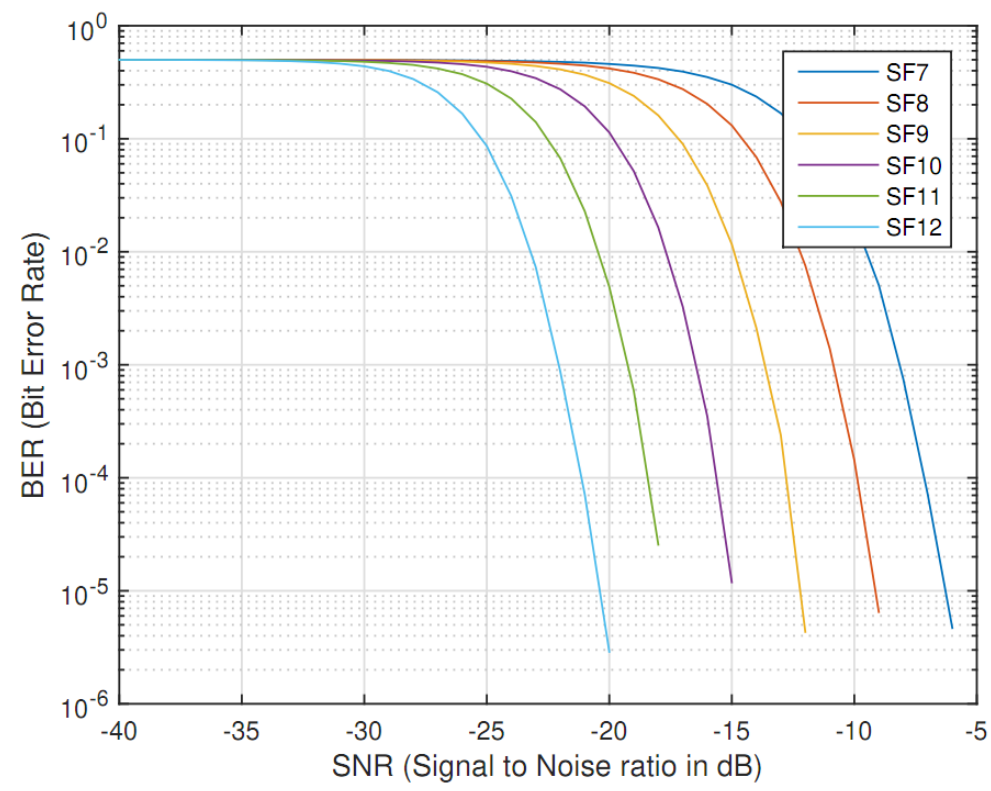

Figure. 7: Plot of SNR vs BER for SF 7 to 12

Spectrograms of packet collisions, for a time span of 60 seconds at a time interval of 10 milliseconds are shown in figure 8. Evaluation is made with 6 channels and 500 devices transmitting simultaneously for spreading factor 7 to 12 . At a bandwidth of $125 \mathrm{kHz}$ at ISM band 
868.7MHz to $869.2 \mathrm{MHz}$, it is observed that packet collisions are higher at $\mathrm{SF}=11$ and 12 compared with lower SFs.

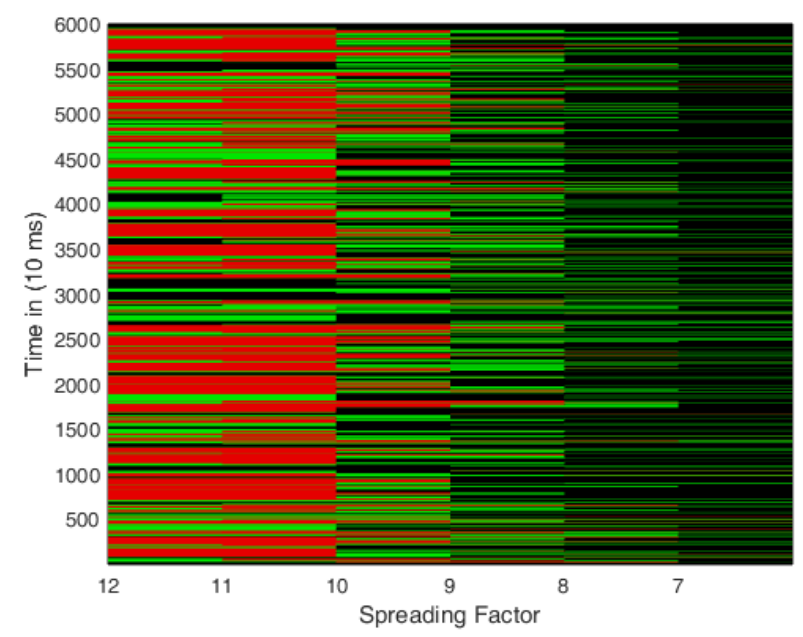

Figure 8. Packet collisions with 500 sensors transmitting randomly for 60 seconds at $125 \mathrm{kHz}$ bandwidth.

Collisions occurred when data packets are transmitted randomly at $10 \mathrm{msec}$ interval with steady increasing packet rate per minute are shown in figure 9. Number of collisions and percentage packet error rate vs number of bytes per minute are plotted.

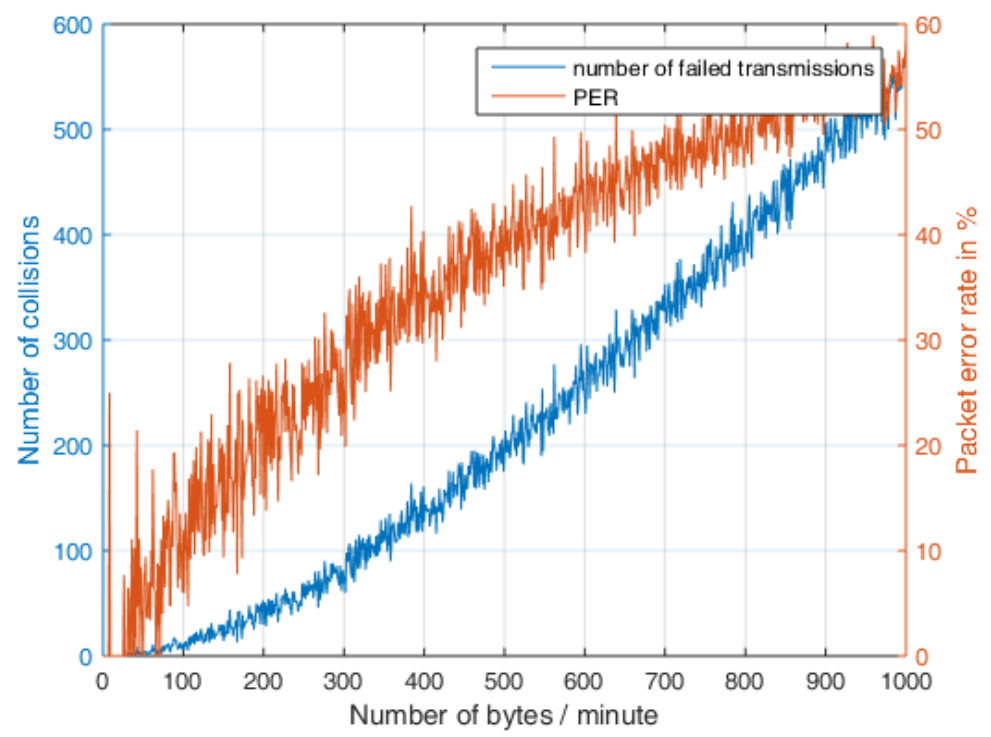

Figure 9. Plot of number of collisions, packet error rate in percentage and number of bytes per minute with 1000 devices transmitting simultaneously with random spreading factor.

The plot of SNR vs. BER for SF 7 to 12 reveals the superior performance of CSS at a high noise level. Acceptable level of BER is observed from $-17 \mathrm{db}$ at $\mathrm{SF}=7$ to $-30 \mathrm{db}$ at $\mathrm{SF}=12$. Extended range can be achieved by enhancing spreading factor as shown in equation 11 but the bit rate is compromised. Adversely when more devices communicate simultaneously at higher packet rate, there are exponential increases in collisions and packet error. An optimum value can be observed as 200 bytes per minute with 1000 device transmit simultaneously. Most of the sensor networks in IoT application needs ultra low bit rate. A data rate of 200 bytes per minute is a substantially 
high value for sensor networks. Even though Chirp Spread Spectrum can efficiently be deployed for setting up ad hoc sensor networks for long-range concerns exists as the number of deployments keeps on increasing, there shall be deterioration in performance due to packet collisions at higher spreading factor. Alternate modulation schemes need to be considered for the massive deployment of LPWAN.

\section{Conclusions}

We analyzed robustness and noise immunity of CSS as a modulation scheme for the deployment of LPWAN. We investigated interference among carriers caused by simultaneous transmissions for collisions. Observations prove better noise immunity of the modulation scheme and suitability for low power wide area sensor networks. Collisions and packet error rate were plotted for 1000 devices transmitting simultaneously at varying packet rate. Simulations proved an exponential increase in collisions and packet error for simultaneous transmissions. Concerns are justified on the deterioration of performance due to massive deployment. The inherent property of chirp spread spectrum as energy efficient modulation scheme together with resilience to noise makes it the best suited for sensor network IoT applications at the ultra-low bit rate. More investigations need to be made to adopt methods to reduce mutual interference that may cause due to massive deployment.

\section{REFERENCES}

[1] Aamir Mahmood, Emiliano Sisinni \& Lakshmikanth Guntupalli,(2018) "Scalability analysis of a LoRa network under imperfect orthogonality", IEEE transactions on industrial informatics(early access), pp1-12.

[2] Marco Centenaro, Lorenzo Vangelista, Andrea Zanella \& Michele Zorzi, (2016) "Long-range communications in unlicensed bands: the rising stars in the IoT and smart city scenarios", IEEE Wireless Communications Journal, Vol. 23, No. 5, pp60-67.

[3] Guillaume Ferr'e, Eric Simon, (2018) "An introduction to Sigfox and LoRa PHY and MAC layers," hal-01774080 pp1-7.

[4] Aiju Thomas \& N.V Eldhose, (2015) "Driver encapsulation and transfer for machine to machine (M2M) communication", International Conference on Control Communication and Computing India (ICCC), vol. 1, pp659-663.

[5] "Ultra Narrow Band radio modulation" Available online: https://www.sigfox.com/en/sigfox-iottechnology-overview (accessed on November 2018).

[6] Ingenu,(2016) “How RPMAWorks -The making of RPMA”, Ingenu inc pp1-92.

[7] A. Springer, W. Gugler, M. Huemer, L. Reindl, C.C.W. Ruppel \& R. Weigel, (2000) "Spread Spectrum Communications Using Chirp Signals," IEEE/AFCEA EUROCOMM 2000. Information Systems for Enhanced Public Safety and Security, Vol. 1, pp166-170.

[8] Application Note, (2015) "LoRa Modulation Basics," SEMTECH Wireless and Sensing, AN1200.22, pp1-26.

[9] SX1272/3/6/7/8: LoRa Modem, (2013) "Designer's Guide" SEMTECH Wireless and Sensing, AN1200. pp1-9. 
[10] Xiaowei Wang, Minrui Fei \& Xin Li,(2008) "Performance of chirp spread spectrum in wireless communication systems," 11th IEEE Singapore International Conference on Communication Systems, Vol 1, pp466-469.

[11] M. Winkler,(1962) "Chirp signals for communications", IEEE WESCON Convention Record, pp7.

[12] A. Berni, W. Gregg, (1973) "On the utility of chirp modulation for digital signalling”, IEEE Transactions on Communications, vol. 21, no. 6, pp748-751.

[13] Andreas F. Molisch, Kannan Balakrishnan, Chia-Chin Chong, Shahriar Emami, Andrew Fort, Johan Karedal, Juergen Kunisch, Hans Schantz, Ulrich Schuster \& Kai Siwiak (2004) "IEEE 802.15.4 a channel model - final report, Molisch ”,pp1-41.

[14] "An overview of the IEEE 802.15.4a Standard" Available on line: https://ieeexplore.ieee.org/ document/5394030 (accessed on October 2018).

[15] T. Smyth,(2013) "Frequency Modulation (FM) Synthesis," in School of Computing Science, Simon Fraser University, pp1-29.

[16] Zhengguo Sheng, Chinmaya Mahapatra,Chunsheng Zhu, Victor C. M \& Leung,(2015) "Recent advances in industrial wireless sensor networks towards efficient management in IoT ," IEEE Access, Vol. 3, pp622-637.

\section{AUTHORS}

Aiju Thomas received his Bachelor of Engineering and Masters of Technology in Electronics and Communication Engineering with specialization in microwave engineering. He is pursuing his research in the optimization of IoT protocols at M G University, Kottayam, Kerala, India. With 10 years of industrial experience and 19 years as faculty in Electronics at Polytechnic institute, his field of interest include industrial and embedded designing.

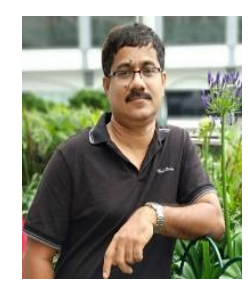

N V Eldhose received his Bachelor and Masters in Science and Ph.D. degree in Physics. As a research guide for M G University, Kerala his research interests include ubiquitous computing, wireless sensor networks, embedded systems, and machine-to-machine communication. He is currently a faculty in Electronics at School of Technology and Applied Science and is guiding eight research projects currently. 